\title{
Association of pathway mutation with survival after recurrence in colorectal cancer patients treated with adjuvant fluoropyrimidine and oxaliplatin chemotherapy
}

Dae-Won Lee ${ }^{1}$, Sae-Won Han ${ }^{1,2^{*}}$ (D, Y Yongjun Cha', Jeong Mo Bae ${ }^{3}$, Hwang-Phill Kim², ${ }^{2,}$ Jaemyun Lyu ${ }^{5}$, Hyojun Han ${ }^{5}$, Hyoki Kim ${ }^{5}$, Hoon Jang ${ }^{6}$, Duhee Bang ${ }^{6}$, Jae-Kyung Won ${ }^{3}$, Seung-Yong Jeong ${ }^{7}$, Kyu Joo Park,

Gyeong Hoon Kang ${ }^{3}$ and Tae-You Kim ${ }^{1,2,4}$

\begin{abstract}
Background: Although the prognostic biomarkers associated with colorectal cancer (CRC) survival are well known, there are limited data on the association between the molecular characteristics and survival after recurrence (SAR). The purpose of this study was to assess the association between pathway mutations and SAR.

Methods: Of the 516 patients with stage III or high risk stage II CRC patients treated with surgery and adjuvant chemotherapy, 87 who had distant recurrence were included in the present study. We analyzed the association between SAR and mutations of 40 genes included in the five critical pathways of CRC (WNT, P53, RTK-RAS, TGF- $\beta$, and PI3K).

Results: Mutation of genes within the WNT, P53, RTK-RAS, TGF- $\beta$, and PI3K pathways were shown in 69(79.3\%), 60(69.0\%), 57(65.5\%), 21(24.1\%), and 19(21.8\%) patients, respectively. Patients with TGF- $\beta$ pathway mutation were younger and had higher incidence of mucinous adenocarcinoma (MAC) histology and microsatellite instability-high. TGF- $\beta$ pathway mutation (median SAR of 21.6 vs. 44.4 months, $p=0.021$ ) and MAC (20.0 vs. 44.4 months, $p=0.003$ ) were associated with poor $S A R$, and receiving curative resection after recurrence was associated with favorable SAR (Not reached vs. 23.6 months, $p<0.001$ ). Mutations in genes within other critical pathways were not associated with SAR. When MAC was excluded as a covariate, multivariate analysis revealed TGF- $\beta$ pathway mutation and curative resection after distant recurrence as an independent prognostic factor for SAR. The impact of TGF- $\beta$ pathway mutations were predicted using the PROVEAN, SIFT, and PolyPhen-2. Among 25 mutations, 23(92.0\%)24(96.0\%) mutations were predicted to be damaging mutation.
\end{abstract}

Conclusions: Mutation in genes within TGF- $\beta$ pathway may have negative prognostic role for SAR in CRC. Other pathway mutations were not associated with SAR.

Keywords: Colorectal cancer, TGF- $\beta$ pathway, Mucinous adenocarcinoma, Survival after recurrence

\footnotetext{
* Correspondence: saewon1@snu.ac.kr

'Department of Internal Medicine, Seoul National University Hospital, 101

Daehang-Ro, Jongno-Gu, Seoul 110-744, South Korea

${ }^{2}$ Cancer Research Institute, Seoul National University College of Medicine,

Seoul, South Korea

Full list of author information is available at the end of the article
}

(c) The Author(s). 2019 Open Access This article is distributed under the terms of the Creative Commons Attribution 4.0 International License (http://creativecommons.org/licenses/by/4.0/), which permits unrestricted use, distribution, and reproduction in any medium, provided you give appropriate credit to the original author(s) and the source, provide a link to the Creative Commons license, and indicate if changes were made. The Creative Commons Public Domain Dedication waiver (http://creativecommons.org/publicdomain/zero/1.0/) applies to the data made available in this article, unless otherwise stated. 


\section{Background}

Colorectal cancer (CRC) is ranked third in cancer incidence and second in cancer-related mortality worldwide [1]. CRC is a heterogeneous disease, and recent data from gene expression profiling have classified CRC into four consensus molecular subtypes (CMSs) [2]. In addition, integrated analysis of somatic mutations, copy number change, and mRNA expression from the Cancer Genome Atlas (TCGA) revealed that the WNT, P53, RTK-RAS, TGF- $\beta$, and PI3K pathways are frequently altered in CRC $[3,4]$.

The current standard care in stage III CRC is complete resection of the tumor followed by adjuvant chemotherapy with 5-fluorouracil, leucovorin, and oxaliplatin (FOLFOX) or capecitabine plus oxaliplatin (XELOX) $[5,6]$. However, approximately $30 \%$ of patients develop recurrence despite receiving adjuvant chemotherapy [7]. Several biomarkers, including microsatellite instability-high (MSI-H), the CpG island methylator phenotype (CIMP), KRAS mutation, and $B R A F$ mutation, are associated with recurrence and survival in CRC patients [8-11]. Although the prognostic biomarkers associated with cancer recurrence are well studied, there is a paucity of data regarding biomarkers associated with survival after recurrence (SAR). Recently, from a study population of stage III CRC patients enrolled in a phase III adjuvant chemotherapy study, MSI-H was associated with improved SAR, and KRAS mutation and $B R A F$ mutation were associated with poor SAR [12].

We have previously reported that mutation in genes within PI3K pathway is associated with better RFS and that mutation in genes within RTK-RAS pathway is associated with worse RFS in CRC treated with adjuvant chemotherapy [13]. The purpose of this study was to evaluate the association between five critical pathway mutations and SAR in CRC patients who had distant recurrence after the treatment of curative surgery followed by adjuvant fluoropyrimidine and oxaliplatin chemotherapy.

\section{Methods}

\section{Patients and treatment}

The study population consisted of patients with stage III or high-risk stage II CRC who developed distant recurrence after receiving curative surgery at Seoul National University Hospital (SNUH, Seoul, South Korea). All patients were included in the CRC patient cohort reported previously [13]. Patients received at least 6 cycles of adjuvant FOLFOX or 4 cycles of adjuvant XELOX chemotherapy. Upper rectal cancer patients were included if they did not receive pre- or post-operative radiation therapy. Exclusion criteria were the following: signet ring cell histology, anti-EGFR or anti-VEGF treatment for adjuvant chemotherapy, history of other malignancy within 5 years, and local recurrence only. Adjuvant FOLFOX was planned for 12 cycles and XELOX chemotherapy was planned for 8 cycles. 50 patients received FOLFOX-4, 27 received FOLFOX-6, and 10 received XELOX. Electronic database and electronic medical record system of SNUH was used to identify eligible patients and review their medical charts.

\section{Molecular testing, including targeted sequencing of 40 genes associated with five critical pathways}

Every exon of the 40 genes associated with the five critical pathways of CRC was sequenced [13]. Fourteen genes were selected from WNT pathway (ARID1A, AMER1, APC, AXIN2, CTNNB1, DKK1, DKK2, DKK3, DKK4, FBXW7, FZD10, LRP5, SOX9, TCF7L2), 2 genes from $\mathrm{P} 53$ pathway (ATM, TP53), 8 genes from RTK-RAS pathway (BRAF, EGFR, ERBB2, ERBB3, ERBB4, HRAS, $K R A S, N R A S), 7$ genes from TGF- $\beta$ pathway $(A C V R 1 B$, ACVR2A, SMAD2, SMAD3, SMAD4, TGFBR1, TGFBR2), and 9 genes from PI3K pathway (IGF1R, IGF2, IRS2, MTOR, PDGFRA, PIK3CA, PIK3R1, PTEN, $S R C)$. Detailed methods for targeted sequencing can be found in our previous article [13]. Briefly, genomic DNA (> $200 \mathrm{ng}$ ) samples were sheared and prepared according to routine library preparation. The captured library was amplified and sequenced using Hiseq 2500 (Illumina, USA). Sequencing data were filtered with a mean quality Q20 (Phred score) per read, and these filtered data were aligned to GRCh37 using bwa 0.7.5a. The aligned reads were processed with Picard Mark Duplicates and GATK base recalibration. After a series of processes, the aligned bases were piled up with SAM tools. Variant call and somatic analysis processes were performed by Varscan and were annotated with ANNOVAR. The pathways were defined as having a mutation if any gene included in the pathway had a mutation.

The microsatellite status was assessed using the following markers: BAT25, BAT26, D2S123, D5S346 and D17S250 [13]. Instability at 2 or more markers were defined as MSI-high, 1 as MSI-low, and 0 as MSS. MSI-H was regarded as having MSI, and MSI-L was grouped with MSS.

\section{Statistical analysis}

The primary objective of this study was to investigate the effect of mutations of critical pathways and clinico-pathologic characteristics on SAR. SAR was defined as the time from distant tumor recurrence to death from any cause. Categorical variables were compared by chi-square test or Fisher's exact test. Continuous variables were compared using the independent-samples $\mathrm{T}$ test. SAR was calculated using the Kaplan-Meier method, and comparisons were made using log-rank tests. Hazard ratios (HRs) were calculated using the Cox proportional hazard model. To adjust for the baseline characteristics, Cox proportional hazard analysis of SAR 
included sex, age (continuous variable), tumor location [proximal (from the cecum to the transverse colon) vs. distal (from the descending colon to the rectum)], tumor histology (mucinous adenocarcinoma vs. non-mucinous adenocarcinoma), tumor ( $\mathrm{T}$ ) stage (continuous variable), lymph node $(\mathrm{N})$ stage (continuous variable), curative operation (done vs. not done), recurrence before 1 year, microsatellite status (MSI-high vs. MSS and MSI-low), and five critical pathway mutations. Two-sided $p$-values less than 0.05 were considered statistically significant. Statistical analysis was performed using SPSS software for Windows, version 20.0 (SPSS, Chicago, IL, USA).

\section{Results}

\section{Patient characteristics}

At a median follow-up duration of 44.4 months, 87 patients had distant recurrence among our database of 516 patients [13]. The baseline characteristics of the 87 patients are summarized in Table 1 . The primary tumor location was proximal (from the cecum to the transverse colon) in 28 patients $(32.2 \%)$ and distal (from the descending colon to the rectum) in 59 patients (67.8\%).
Nine patients (10.3\%) had MAC histology, and 3 patients (3.5\%) showed MSI-H feature.

After distant recurrence, palliative chemotherapy was introduced in 77 (88.5\%) patients. The first-line chemotherapy regimens included FOLFIRI in 56 patients, capecitabine monotherapy in 8 , FOLFIRI plus bevacizumab in 7, FOLFIRI plus cetuximab in 2, FOLFOX in 2, S-1 plus oxaliplatin in 1 , and capecitabine plus irinotecan in 1. Second-line chemotherapy was administered to 47 (54.0\%) patients. Only 1 patient received both bevacizumab and cetuximab during the course of treatment. In total, $9(10.3 \%)$ patients received bevacizumab and 9 (10.3\%) patients received cetuximab during their course of treatment.

\section{Gene mutation and pathway mutation}

In patients with recurrence, mutations were most frequently found in TP53 (65.5\%) followed by APC (64.4\%), KRAS (48.3\%), FBXW7 (16.1\%), and SMAD4 (10.3\%) (Additional file 1: Table S1). The WNT pathway was mutated in $79.3 \%$, P53 in $69.0 \%$, RTK-RAS in $65.5 \%$, TGF- $\beta$ in $24.1 \%$, and PI3K in $21.8 \%$ of patients. The mutation frequencies of most genes were similar between

Table 1 Baseline characteristics

\begin{tabular}{|c|c|c|c|c|c|}
\hline Characteristics & & $\begin{array}{l}\text { Total } \\
(N=87)\end{array}$ & $\begin{array}{l}\text { TGF- } \beta \text { WT } \\
(N=66)\end{array}$ & $\begin{array}{l}\text { TGF- } \beta \text { MT } \\
(N=21)\end{array}$ & P-Value \\
\hline Age & Median (range) & $59(31-75)$ & $61(38-75)$ & $54(31-74)$ & 0.020 \\
\hline \multirow[t]{2}{*}{ Sex } & Male & $50(57.5 \%)$ & $36(54.4 \%)$ & $14(66.7 \%)$ & 0.33 \\
\hline & Female & 37 (42.5\%) & $30(45.5 \%)$ & $7(33.3 \%)$ & \\
\hline \multirow[t]{2}{*}{ Location } & Proximal & $28(32.2 \%)$ & 19 (28.8\%) & $9(42.9 \%)$ & 0.23 \\
\hline & Distal & 59 (67.8\%) & $47(71.2 \%)$ & 12 (57.1\%) & \\
\hline \multirow[t]{2}{*}{ Stage } & II, high-risk & $14(16.1 \%)$ & $8(12.1 \%)$ & $6(28.6 \%)$ & 0.074 \\
\hline & III & $73(83.9 \%)$ & $58(87.9 \%)$ & $15(71.4 \%)$ & \\
\hline \multirow[t]{4}{*}{ T stage } & 1 & $0(0.0 \%)$ & $0(0.0 \%)$ & $0(0.0 \%)$ & 0.41 \\
\hline & 2 & $1(1.1 \%)$ & $1(1.5 \%)$ & $0(0.0 \%)$ & \\
\hline & 3 & $62(71.3 \%)$ & 49 (74.2\%) & $13(61.9 \%)$ & \\
\hline & 4 & $24(27.6 \%)$ & $16(24.2 \%)$ & $8(38.1 \%)$ & \\
\hline \multirow[t]{3}{*}{ N stage } & 0 & $14(16.1 \%)$ & $8(12.1 \%)$ & $6(28.6 \%)$ & 0.18 \\
\hline & 1 & $29(33.3 \%)$ & $24(36.4 \%)$ & $5(23.8 \%)$ & \\
\hline & 2 & $44(50.6 \%)$ & $34(51.5 \%)$ & $10(47.6 \%)$ & \\
\hline \multirow[t]{2}{*}{ Histology } & Non-MAC & 78 (89.7\%) & $65(98.5 \%)$ & $13(61.9 \%)$ & $<0.001$ \\
\hline & MAC & $9(10.3 \%)$ & $1(1.5 \%)$ & $8(38.1 \%)$ & \\
\hline \multirow[t]{2}{*}{ MSI } & MSS/MSI-L & $83(96.5 \%)$ & $65(100.0 \%)$ & $18(85.7 \%)$ & 0.013 \\
\hline & MSI-H & $3(3.5 \%)$ & $0(0.0 \%)$ & $3(14.3 \%)$ & \\
\hline \multirow[t]{2}{*}{ Time to recurrence } & $<1$ year & $19(21.8 \%)$ & $11(16.7 \%)$ & $8(38.1 \%)$ & 0.038 \\
\hline & $\geq 1$ year & $68(78.2 \%)$ & $55(83.3 \%)$ & $13(61.9 \%)$ & \\
\hline \multirow[t]{2}{*}{ Curative resection after distant recurrence } & Not done & $52(59.8 \%)$ & $39(59.1 \%)$ & $13(61.9 \%)$ & 0.82 \\
\hline & Done & $35(40.2 \%)$ & $27(40.9 \%)$ & $8(38.1 \%)$ & \\
\hline
\end{tabular}


the patients with recurrence and without recurrence (Additional file 1: Table S1). The mutation frequency of PIK3CA and CTNNB1 was lower in the patients with recurrence, and $A P C$ showed a tendency of lower frequency in patients with recurrence. PI3K pathway mutation showed a tendency of lower frequency in patients with recurrence.

Patients with mutation in genes within TGF- $\beta$ pathway showed distinct characteristics compared with patients without TGF- $\beta$ pathway mutation (Table 1). Patients with TGF- $\beta$ pathway mutation was associated with a younger age (median age of 54 years vs. 61 years, $p=$ 0.020 ), and there was a tendency of a higher percentage of patients with initial high-risk stage II disease $(28.6 \%$ vs. $12.1 \%, p=0.074)$. Moreover, patients with TGF- $\beta$ pathway mutation had a higher incidence of MSI-H and MAC. All 3 patients with recurrence in MSI-H had TGF- $\beta$ pathway mutation. In addition, the incidence of TGF- $\beta$ pathway mutation was $88.9 \%$ (8/9) in MAC patients compared with $16.7 \%(13 / 78)$ in non-MAC patients $(p<0.001)$ (Additional file 1: Table S2). The mutation incidences of other pathways were similar between MAC and non-MAC histology (Additional file 1: Table S2).

\section{Prognostic role of five critical pathways}

Among the 87 patients who had distant recurrence, 40 death events occurred with an estimated median SAR of 38.2 months. SAR was significantly worse in patients with TGF- $\beta$ pathway mutation than in those with wild type (median SAR of 21.6 vs. 44.4 months, $p=0.021$ ) (Fig. 1). In our previous report, mutation in genes within PI3K pathway was associated with a longer RFS, whereas

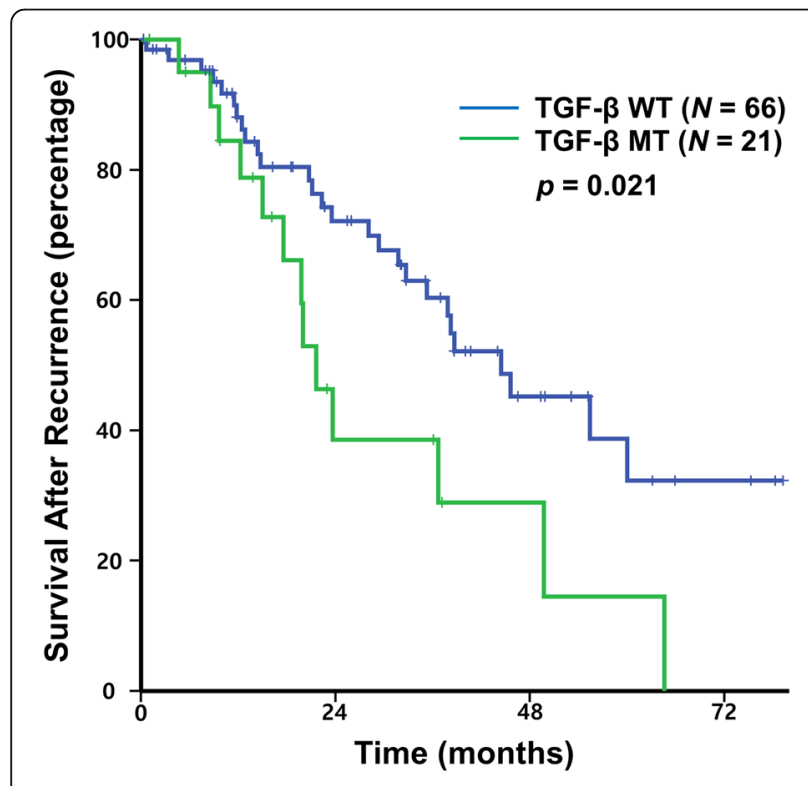

Fig. 1 TGF- $\beta$ pathway mutation and survival after recurrence mutation in genes within RTK-RAS pathway was associated with a shorter RFS [13]. However, there was no correlation between SAR and other critical pathway mutations (Additional file 2: Figure S1). In addition to TGF- $\beta$ pathway mutation, curative resection after distant recurrence and MAC was associated with poor SAR, and N2 stage showed a tendency of poor SAR (Table 2). Tumor location and MSI were not associated with SAR. Multivariate analysis using the Cox proportional hazard model revealed that curative resection after distant recurrence [adj HR for SAR 0.27 (95\% CI 0.13-0.56), $p=$ 0.001] and MAC histology [adj HR for SAR 3.77 (95\% CI 1.52-9.35), $p=0.004$ ] were independent prognostic factors for SAR. In the present study, MAC histology showed a higher incidence of TGF- $\beta$ pathway mutation compared to non-MAC histology (88.9\% vs. $16.7 \%, p<0.001$ ) (Additional file 1: Table S2). Although statistically not significant, the negative prognostic role of TGF- $\beta$ pathway mutation was maintained in patients with non-MAC histology (median SAR of 36.7 vs. 44.4 months, $p=0.127$ ) (Fig. 2). After removing MAC histology as a covariate, multivariate analysis revealed TGF- $\beta$ pathway mutation [adj HR for SAR 1.99 (95\% CI 1.02-3.90), $p=0.044$ ] and curative resection after distant recurrence [adj HR for SAR 0.28 (95\% CI 0.13-0.59), $p=0.001$ ] as independent prognostic factors for SAR.

We next analyzed whether individual gene mutation is associated with SAR (Additional file 1: Table S2). Patients with SMAD4 mutation had worse SAR compared to wild type (SAR of 17.6 months vs. 38.7 months, $p=0031$ ) and those with $A P C$ mutation had a tendency of favorable SAR compared to wild type (SAR of 45.6 months vs. 28.1 months, $p=0070$ ). In the multivariate analysis, SMAD4 mutation was an independent negative prognostic factor for SAR [adjusted hazard ratio (adj HR) for SAR 3.54 (95\% CI 1.13-11.03), $p=0.030]$. The detailed mutation profile of TGF- $\beta$ pathway is shown in Table S4 (Additional file 1: Table S4). Among 25 mutations, 5 were stop gain mutation, 4 were frame shift deletion mutation, and 2 were non-frameshift deletion mutation which would probably lead to loss of expression. Impact of 14 non-synonymous SNV in TGF- $\beta$ pathway mutation was predicted using the PROVEAN, SIFT, and PolyPhen-2 (Additional file 1: Table S2) [14-16]. Among 14 non-synonymous SNV, 12(85.7\%)13(92.9\%) were predicted to be damaging mutation (Additional file 1: Table S4). In total, 23(92.0\%)$24(96.0 \%)$ mutations were predicted to be damaging mutation in the TGF- $\beta$ pathway. We also predicted functional outcome of SMAD4 mutations using the OncoKB database (Additional file 1: Table S4) [17]. Among 9 SMAD4 mutations, 3 mutations were 
Table 2 Univariate analysis of survival after recurrence

\begin{tabular}{|c|c|c|c|}
\hline Variable & & Median SAR (months) & $p$-value \\
\hline \multirow[t]{2}{*}{ Age } & $<65$ years & $36.7(26.0-47.3)$ & 0.71 \\
\hline & $\geq 65$ years & $45.6(29.4-61.8)$ & \\
\hline \multirow[t]{2}{*}{ Sex } & Male & $37.8(33.6-42.1)$ & 0.49 \\
\hline & Female & $55.4(0.0-110.8)$ & \\
\hline \multirow[t]{2}{*}{ Location } & Proximal & $37.8(13.9-61.8)$ & 0.82 \\
\hline & Distal & $38.7(27.9-49.4)$ & \\
\hline \multirow[t]{2}{*}{ Time to recurrence } & $<1$ year & $31.7(5.9-57.6)$ & 0.16 \\
\hline & $\geq 1$ year & $44.4(31.4-57.4)$ & \\
\hline \multirow[t]{2}{*}{ Stage } & II, high risk & $49.7(\mathrm{~N} / \mathrm{A})$ & 0.36 \\
\hline & III & $37.8(30.2-45.5)$ & \\
\hline \multirow[t]{2}{*}{ T-stage } & $\mathrm{T} 1-3$ & $38.2(29.0-47.4)$ & 0.31 \\
\hline & $\mathrm{T} 4$ & $28.1(0.0-64.8)$ & \\
\hline \multirow[t]{2}{*}{ N-stage } & No-1 & $49.7(26.1-73.3)$ & 0.064 \\
\hline & N2 & $29.3(11.4-47.3)$ & \\
\hline \multirow[t]{2}{*}{ Histology } & Non-MAC & $44.4(31.2-54.7)$ & 0.003 \\
\hline & MAC & $20.0(4.9-35.0)$ & \\
\hline \multirow[t]{2}{*}{ Curative resection after distant recurrence } & Not done & $23.6(12.1-35.2)$ & $<0.001$ \\
\hline & Done & Not reached & \\
\hline \multirow[t]{2}{*}{ MSI } & MSS/MSI-L & $38.2(28.2-48.2)$ & 0.35 \\
\hline & MSI-H & $9.3(1.7-38.2)$ & \\
\hline
\end{tabular}

Abbreviations: MAC mucinous adenocarcinoma, MSS microsatellite stable, MSI-L microsatellite instability-low, MSI-H microsatellite instability-high, N/A not available

found to be loss-of-function and additional 2 mutations involved the same location with aforementioned loss-of-function mutation. $A C V R 1 B$ and $A C V R 2 A$ genes were not covered by the OncoKB and our mutation involving SMAD2, SMAD3,

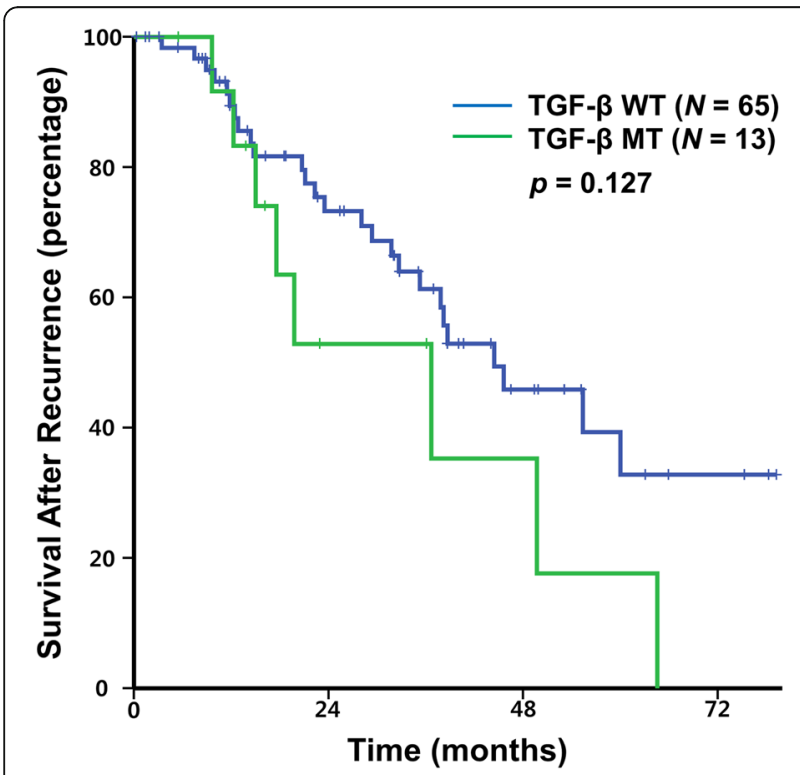

Fig. 2 TGF- $\beta$ pathway mutation and survival after recurrence in Non-MAC patients. Abbreviations: MAC, mucinous adenocarcinoma
TGFBR1, and TGFBR2 was not reported in the OncoKB.

\section{Discussion}

This study revealed that mutation in genes within TGF- $\beta$ pathway is associated with MAC and may have poor SAR in colorectal cancer patients. Other critical pathways were not associated with SAR. In our previous study, mutation in genes within TGF- $\beta$ pathway was not associated with RFS [13]. This implicates that while TGF- $\beta$ pathway mutation is not associated with recurrence in early-stage tumors, it may be fatal in later-stage tumors.

In the present study, patients with TGF- $\beta$ pathway mutation had higher incidence of MAC. This is in line with data from the TCGA where TGF- $\beta$ pathway is more frequently altered in MAC histology compared to non-MAC histology $(67.9 \%$ vs. $30.6 \%, p<0.001)$ [18]. The TGF- $\beta$ pathway is associated with cell proliferation, cell differentiation, apoptosis, and epithelial-mesenchymal transition (EMT) [19, 20]. Although mutational inactivation of TGF$\beta$ pathway is frequently shown in colorectal cancer, the level of TGF-b production is somehow elevated in later stage tumor [21, 22]. One of the reasons is that TGF-b level is not only affected by tumor but is also affected by stromal cells $[22,23]$. The correlation between TGF- $\beta$ pathway mutation (which is usually loss-of function) and TGF-b level has not been clearly identified and maybe 
different among disease status. In terms of clinical relevance, there are reports showing that TGF- $\beta$ pathway may have a prognostic role in CRC. CMS classifies colorectal cancer based on the gene expression profile [2]. CMS4, which is characterized by the upregulation of genes implicated in EMT, prominent TGF- $\beta$ pathway activation, stromal invasion, and angiogenesis, is associated with a worse prognosis. In addition, preclinical studies show that activation of TGF- $\beta$ pathway may be linked to chemotherapy resistance in CRC cell line $[24,25]$. Recombinant TGF- $\beta$ treatment caused chemotherapy resistance in CRC cell lines [24]. However, TGF- $\beta$ pathway mutation was not associated with RFS in CRC patients treated with adjuvant FOLFOX/XELOX chemotherapy, which we have previously reported [13]. Discordance in the prognostic role of TGF- $\beta$ pathway mutation among disease status could be partially explained by the fact that the response to TGF- $\beta$ pathway activation is different according to cell type and status $[20,26]$. There are opposite effects of the TGF- $\beta$ pathway in that it may suppress the growth of tubular adenoma but may promote the tumor growth of sessile serrated adenomas by inducing EMT and metastasis [20, 26]. Apoptosis is the dominant feature after TGF- $\beta$ pathway stimulation in classical tubular adenoma organoid culture [26]. By contrast, inducing EMT is the main outcome after TGF- $\beta$ pathway stimulation in an organoid culture model for sessile serrated adenomas [26]. The activation of TGF- $\beta$ may suppress the growth of tubular adenoma but may progress sessile serrated adenomas to CMS4 subtype cancer by inducing EMT [26]. Moreover, evidence show that TGF-b level is also affected by stromal cells [22]. While TGF- $\beta$ pathway mutation is not associated with RFS in CRC patients treated with adjuvant FOLFOX/ XELOX chemotherapy, it may be fatal in CRC patients who have recurred after curative surgery. Among the genes involved in the TGF- $\beta$ pathway, SMAD4 mutation was associated with poor SAR. SMAD4 is a tumor suppressor gene and its mutation is involved in advanced stages, such as distant metastasis, in human colorectal carcinogenesis [27]. In the present study, we predicted outcome of TGF- $\beta$ pathway mutation using the PROVEAN, SIFT, and PolyPhen-2. Among 14 non-synonymous SNV in TGF- $\beta$ pathway mutation $12(85.7 \%)-13(92.9 \%)$ were predicted to be damaging mutation. Other 11 mutations consisted of 5 stop gain mutation, 4 frame shift deletion mutation, and 2 non-frameshift deletion mutation which would probably lead to loss of expression. In sum, 23(92.0\%)-24(96.0\%) mutations were predicted to be damaging mutation in the TGF- $\beta$ pathway. In addition, all SMAD4 mutations reported in the OncoKB database were loss-of-function mutation. This shows that TGF- $\beta$ pathway mutation may have contributed to poor SAR due to loss of function. The correlation between TGF- $\beta$ pathway mutation,
TGF-b expression, and chemotherapy resistance needs to be studied in the future.

Sinicrope et al. reported that MSI-H is associated with improved SAR and that KRAS mutation and BRAF mutation are associated with poor SAR in stage III colon cancer patients treated with adjuvant chemotherapy [12]. However, we could not evaluate the role of MSI-H and $B R A F$ mutation in SAR because there were only 3 patients with MSI-H and 5 patients with $B R A F$ V600E mutation. In contrast to a study by Sinicrope et al., RTK-RAS pathway mutation and KRAS exon 2 mutation were not associated with SAR in the present study. In a study by Sinicrope et al., the negative prognostic role of KRAS mutation was limited to patients who received adjuvant FOLFOX pus cetuximab but not in patients treated with adjuvant FOLFOX alone. In the present study, none of the patients received cetuximab in the adjuvant setting. This implicates that KRAS mutation may not affect SAR in patients treated with adjuvant FOLFOX or XELOX alone.

The major limitation of this study was that we did not evaluate genetic characteristics other than targeted sequencing of genes associated with 5 critical pathways. Although major genes included in the TGF- $\beta$ pathway were sequenced, other alterations, including copy number variation or the expression level, were not analyzed. It is also important to analyze the TGF- $\beta$ pathway induced by stromal cells $[3,23]$. Therefore, comprehensive analysis of the TGF- $\beta$ pathway could provide a better understanding of CRC biology in the future. Another limitation of the present study was that patients were treated differently after recurrence. However, this study was performed at a high-volume single center where the treatment plan was relatively uniform. The last limitation was the relatively small number of patients included in this cohort. However, this was the first study to evaluate the relationship between pathway mutation and SAR in CRC patients.

\section{Conclusions}

This study revealed that CRC patients with mutation in genes within TGF- $\beta$ pathway may have poor SAR. Other pathway mutations were not associated with SAR. Future works on the relationship between TGF- $\beta$ pathway mutation and survival according to disease status needs to be studied.

\section{Additional files}

Additional file 1: Table S1. Mutation rate of 40 genes included in the study. Table S2. Mutation rate of critical pathways according to histology. Table S3. Survival after recurrence according to each gene mutation. (Genes with mutation rate over 5\%). Table S4. Detailed profile of TGF- $\beta$ pathway mutation. (DOCX $60 \mathrm{~kb}$ )

Additional file 2: Figure S1. Pathway mutations and survival after recurrence. Mutation in pathways other than TGF- $\beta$ were not associated with survival after recurrence. (TIF $311 \mathrm{~kb}$ ) 


\section{Abbreviations}

Cl: Confidence interval; CIMP: CpG island methylator phenotype; CMS: Consensus molecular subtypes; CRC: Colorectal cancer; EMT: Epithelialmesenchymal transition; FOLFOX: 5-fluorouracilleucovorin, and oxaliplatin; HR: Hazard ratio; MAC: Mucinous adenocarcinoma; MSI: Microsatellite instability; MSS: Microsatellite-stable; OS: Overall survival; RFS: Relapse-free survival; SAR: Survival after recurrence; SNUH: Seoul National University Hospital; TCGA: The Cancer Genome Atlas; XELOX: Capecitabine plus oxaliplatin

\section{Acknowledgements}

Not applicable.

\section{Funding}

This research was supported by the Seoul National University Hospital (SNUH) Research Fund (03-2014-0440) and a grant of the Korea Health Technology R\&D Project through the Korea Health Industry Development Institute (KHIDI), funded by the Ministry of Health \& Welfare, Republic of Korea (grant number: HI14C1277 and H113C2163). The funding bodies had no influence on the design of the study and collection, analysis, and interpretation of data and in writing the manuscript.

\section{Availability of data and materials}

The datasets used and/or analyzed during the current study are available from the corresponding author on reasonable request.

\section{Authors' contributions}

DWL, SWH and TYK were involved in study concept and design. JMB, JKW, SYJ, KJP, GHK and TYK involved in data acquisition. Analysis and interpretation of data was done by YC, HPK, JL, HH, HK, HJ, DB, and SWH. Manuscript writing was made by DWL, SWH and TYK. Manuscript review and final approval was done by and SWH, JMB, JKW, SYJ, KJP, GHK and TYK. All authors read and approved the final manuscript.

\section{Ethics approval and consent to participate}

The study protocol was reviewed and approved by the institutional review board of SNUH [H-1210-016-430]. This study was carried out in accordance with the recommendations of the Declaration of Helsinki for biomedical research involving human subjects. In accordance with South Korea regulation, retrospective studies without any additional therapy or monitoring procedure, do not need formal written consent from patients. Because the study was retrospective designed without any investigational intervention, the study-specific informed consent was not obtained from each patient. (https://cris.snuh.org, http://bri.snuh.org).

\section{Consent for publication}

Not applicable.

\section{Competing interests}

The authors declare that they have no competing interests.

\section{Publisher's Note}

Springer Nature remains neutral with regard to jurisdictional claims in published maps and institutional affiliations.

\footnotetext{
Author details

'Department of Internal Medicine, Seoul National University Hospital, 101 Daehang-Ro, Jongno-Gu, Seoul 110-744, South Korea. ${ }^{2}$ Cancer Research Institute, Seoul National University College of Medicine, Seoul, South Korea. ${ }^{3}$ Department of Pathology, Seoul National University College of Medicine, Seoul, South Korea. ${ }^{4}$ Department of Molecular Medicine \& Biopharmaceutical Sciences, Graduate School of Convergence Science and Technology, Seoul National University, Seoul, South Korea. ${ }^{5}$ Celemics Inc, Seoul, South Korea. ${ }^{6}$ Department of Chemistry College of Science, Yonsei University, Seoul, South Korea. ${ }^{7}$ Department of Surgery, Seoul National University Hospital, Seoul, South Korea.
}

Received: 5 March 2019 Accepted: 26 April 2019

Published online: 06 May 2019

\section{References}

1. Jemal A, Bray F, Center MM, Ferlay J, Ward E, Forman D. Global cancer statistics. CA Cancer J Clin. 2011;61:69-90.

2. Guinney J, Dienstmann R, Wang X, de Reynies A, Schlicker A, Soneson C, Marisa L, Roepman P, Nyamundanda G, Angelino P, et al. The consensus molecular subtypes of colorectal cancer. Nat Med. 2015;21:1350-6.

3. Cancer Genome Atlas N. Comprehensive molecular characterization of human colon and rectal cancer. Nature. 2012;487:330-7.

4. Fearon ER. Molecular genetics of colorectal cancer. Annu Rev Pathol. 2011;6: 479-507.

5. Andre T, Boni C, Navarro M, Tabernero J, Hickish T, Topham C, Bonetti A, Clingan P, Bridgewater J, Rivera F, et al. Improved overall survival with oxaliplatin, fluorouracil, and leucovorin as adjuvant treatment in stage II or III colon cancer in the MOSAIC trial. J Clin Oncol. 2009;27:3109-16.

6. Haller DG, Tabernero J, Maroun J, de Braud F, Price T, Van Cutsem E, Hill M, Gilberg F, Rittweger K, Schmoll HJ. Capecitabine plus oxaliplatin compared with fluorouracil and folinic acid as adjuvant therapy for stage III colon cancer. J Clin Oncol. 2011;29:1465-71.

7. Shi Q, Andre T, Grothey A, Yothers G, Hamilton SR, Bot BM, Haller DG, Van Cutsem E, Twelves C, Benedetti JK, et al. Comparison of outcomes after fluorouracil-based adjuvant therapy for stages II and III colon cancer between 1978 to 1995 and 1996 to 2007: evidence of stage migration from the ACCENT database. J Clin Oncol. 2013:31:3656-63.

8. Roth AD, Tejpar S, Delorenzi M, Yan P, Fiocca R, Klingbiel D, Dietrich D, Biesmans B, Bodoky G, Barone C, et al. Prognostic role of KRAS and BRAF in stage II and III resected colon cancer: results of the translational study on the PETACC-3, EORTC 40993, SAKK 60-00 trial. J Clin Oncol. 2010;28:466-74.

9. Popat $\mathrm{S}$, Hubner R, Houlston RS. Systematic review of microsatellite instability and colorectal cancer prognosis. J Clin Oncol. 2005;23:609-18.

10. Van Rijnsoever M, Elsaleh H, Joseph D, McCaul K, lacopetta B. CpG island methylator phenotype is an independent predictor of survival benefit from 5-fluorouracil in stage III colorectal cancer. Clin Cancer Res. 2003;9:2898-903.

11. Sinicrope FA, Mahoney MR, Smyrk TC, Thibodeau SN, Warren RS, Bertagnolli MM, Nelson GD, Goldberg RM, Sargent DJ, Alberts SR. Prognostic impact of deficient DNA mismatch repair in patients with stage III colon cancer from a randomized trial of FOLFOX-based adjuvant chemotherapy. J Clin Oncol. 2013;31:3664-72.

12. Sinicrope FA, Shi Q, Allegra CJ, Smyrk TC, Thibodeau SN, Goldberg RM, Meyers JP, Pogue-Geile KL, Yothers G, Sargent DJ, et al. Association of DNA mismatch repair and mutations in BRAF and KRAS with survival after recurrence in stage III Colon cancers : a secondary analysis of 2 randomized clinical trials. JAMA Oncol. 2017;3:472-80.

13. Lee DW, Han SW, Cha Y, Bae JM, Kim HP, Lyu J, Han H, Kim H, Jang H, Bang $D$, et al. Association between mutations of critical pathway genes and survival outcomes according to the tumor location in colorectal cancer. Cancer. 2017;123:3513-23.

14. Choi Y, Chan AP. PROVEAN web server: a tool to predict the functional effect of amino acid substitutions and indels. Bioinformatics. 2015;31:2745-7.

15. Sim NL, Kumar P, Hu J, Henikoff S, Schneider G, Ng PC. SIFT web server: predicting effects of amino acid substitutions on proteins. Nucleic Acids Res. 2012;40:W452-7.

16. Adzhubei IA, Schmidt S, Peshkin L, Ramensky VE, Gerasimova A, Bork P, Kondrashov AS, Sunyaev SR. A method and server for predicting damaging missense mutations. Nat Methods. 2010;7:248-9.

17. Chakravarty D, Gao J, Phillips SM, Kundra R, Zhang H, Wang J, Rudolph JE, Yaeger R, Soumerai T, Nissan MH, et al. OncoKB: a precision oncology Knowledge Base. JCO Precis Oncol. 2017;2017.

18. TCGA. Comprehensive molecular characterization of human colon and rectal cancer. Nature. 2012;487:330-7.

19. Jung B, Staudacher JJ, Beauchamp D. Transforming growth factor beta superfamily signaling in development of colorectal Cancer. Gastroenterology. 2017;152:36-52.

20. Massague J. TGFbeta signalling in context. Nat Rev Mol Cell Biol. 2012;13: 616-30.

21. Langenskiold M, Holmdahl L, Falk P, Angenete E, Ivarsson ML. Increased TGF-beta 1 protein expression in patients with advanced colorectal cancer. J Surg Oncol. 2008;97:409-15. 
22. Calon A, Espinet E, Palomo-Ponce S, Tauriello DV, Iglesias M, Cespedes MV, Sevillano M, Nadal C, Jung P, Zhang XH, et al. Dependency of colorectal cancer on a TGF-beta-driven program in stromal cells for metastasis initiation. Cancer Cell. 2012;22:571-84.

23. Calon A, Lonardo E, Berenguer-Llergo A, Espinet E, Hernando-Momblona X, Iglesias M, Sevillano M, Palomo-Ponce S, Tauriello DV, Byrom D, et al. Stromal gene expression defines poor-prognosis subtypes in colorectal cancer. Nat Genet. 2015;47:320-9.

24. Huang S, Holzel M, Knijnenburg T, Schlicker A, Roepman P, McDermott U, Garnett M, Grernrum W, Sun C, Prahallad A, et al. MED12 controls the response to multiple cancer drugs through regulation of TGF-beta receptor signaling. Cell. 2012;151:937-50.

25. Brunen D, Willems SM, Kellner U, Midgley R, Simon I, Bernards R. TGF-beta: an emerging player in drug resistance. Cell Cycle. 2013;12:2960-8.

26. Fessler E, Drost J, van Hooff SR, Linnekamp JF, Wang X, Jansen M, De Sousa EMF, Prasetyanti PR, JE IJ, Franitza M, et al. TGFbeta signaling directs serrated adenomas to the mesenchymal colorectal cancer subtype. EMBO Mol Med. 2016;8:745-60.

27. Miyaki M, lijima T, Konishi M, Sakai K, Ishii A, Yasuno M, Hishima T, Koike M, Shitara N, Iwama T, et al. Higher frequency of Smad4 gene mutation in human colorectal cancer with distant metastasis. Oncogene. 1999;18:3098-103.

Ready to submit your research? Choose BMC and benefit from:

- fast, convenient online submission

- thorough peer review by experienced researchers in your field

- rapid publication on acceptance

- support for research data, including large and complex data types

- gold Open Access which fosters wider collaboration and increased citations

- maximum visibility for your research: over $100 \mathrm{M}$ website views per year

At $\mathrm{BMC}$, research is always in progress.

Learn more biomedcentral.com/submissions 